\title{
Many-body spectrum and particle localization in quantum dots and finite rotating bose condensates
}

\author{
M. Manninen ${ }^{1}$, S. Viefers ${ }^{2}$, M. Koskinen ${ }^{1}$ and S.M. Reimann ${ }^{3}$ \\ ${ }^{1}$ Department of Physics, University of Jyväskylä, FIN-40351 Jyväskylä, Finland \\ ${ }^{2}$ Department of Applied Physics, Chalmers University of Technology, S-41296 Gothenburg, \\ Sweden \\ ${ }^{3}$ Mathematical Physics, Lund Institute of Technology, P.O. Box 118, 22100 Lund, Sweden
}

(November 13, 2018)

\begin{abstract}
The yrast spectra (i.e. the lowest states for a given total angular momentum) of quantum dots in strong magnetic fields, are studied in terms of exact numerical diagonalization and analytic trial wave functions. We argue that certain features (cusps) in the many-body spectrum can be understood in terms of particle localization due to the strong field. A new class of trial wavefunctions supports the picture of the electrons being localized in Wigner molecule-like states consisting of consecutive rings of electrons, with low-lying excitations corresponding to rigid rotation of the outer ring of electrons. The geometry of the Wigner molecule is independent of interparticle interactions and the statistics of the particles.
\end{abstract}

PACS: 67.40.Db, 73.21.La 


\section{INTRODUCTION}

In a sufficiently strong magnetic field, the spin degree of freedom of electrons in quantum dots is frozen out, and they may be treated as spinless. Furthermore, if the electron density is low, the strong field will cause localization of the electrons in a Wigner molecule (a finite size realization of a Wigner crystal). For a circular dot, this localization happens gradually as the magnetic field increases and is first seen as a separation of a ring of electrons [1,2] from the so-called maximum density droplet (MDD) [3, [1], the finite-size analog of the $\nu=1$ quantum Hall [5] state with angular momentum $N(N-1) / 2$. By increasing the field further, eventually all the electrons localize [6] as in a classical one-component plasma. This localization takes place in the internal coordinate system of the dot and may be revealed by using a rotating frame or studying pair correlation functions [7].

In this paper we study the interplay between particle localization in a finite 2D electron system in a harmonic confinement and the structure of its yrast spectrum, i.e. its lowestlying many-body states as function of the total angular momentum $L$. Several classes of trial many-body wavefunctions are available to describe the yrast states of such a system. In particular, electron states with angular momenta equal to odd multiples of $N(N-1) / 2$ can be approximately described by the Laughlin wavefunction for the fractional Hall effect [8]. Another class of wavefunctions that has proven successful in describing yrast states away from these special $L$-values [9, 10] is due to Jain [11].

Here, we introduce an additional type of trial wavefunctions which, although perhaps not as accurate as the Jain construction, in our opinion give a more intuitive picture of the structure of the ground state of the dot as one goes from a Laughlin state, $L=q N(N-1) / 2$ to the quasihole- or single vortex state $L=q N(N-1) / 2+N$. These wavefunctions are similar to those recently introduced by Bertsch and Papenbrock (BP) [12 to describe the ground state of a weakly repulsive, harmonically trapped, rotating 3D Bose condensate for $2 \leq L \leq N$. The close analogy between this system and a fermionic quantum dot in the quantum Hall regime has been discussed in a number of recent papers [13 16]; the essential observation is that the weak interaction limit allows for a two-dimensional description of the boson system in terms of lowest Landau level (LLL) wavefunctions which, just as for the quantum Hall effect, may contain powers of the Laughlin-Jastrow factor $\prod_{i<j}^{N}\left(z_{i}-z_{j}\right)$, with $z_{i}$ the complex coordinate of the $i$ th particle. The only difference is the symmetry of the many-body wavefunction. This means that it is possible to 'borrow' techniques familiar from quantum Hall physics to describe the rotating Bose condensates and vice versa.

The outline of the paper is as follows. In section 2 we present exact numerical calculations for our test case, the six-electron dot. Trial wavefunctions are introduced and compared to the exact results in section 3. Finally, we discuss the many-body spectra in terms of electron localization, using both our numerical and analytical results.

\section{EXACT CI CALCULATIONS AND THE YRAST SPECTRA}

Independent electrons in a strong magnetic field occupy Fock-Darwin states [17, 18] with

the effective confinement potential determined by the frequency $\omega_{\text {eff }}=\sqrt{\omega_{0}^{2}+\omega_{c}^{2} / 4}$, where $\omega_{0}$ is the external confinement and $\omega_{c}$ the cyclotron frequency $e B / m$. In the absence of 
interactions, the ground- and low-lying excited states (for high angular momentum values) are entirely in the lowest Landau level (LLL).

In order to solve the many-body problem in the presence of Coulomb interactions, we use the configuration interaction technique (CI); it has the advantage that the many-body energy spectrum, not only the ground state, can be computed and that in the case of small particle numbers the solution is essentially exact. We choose a six electron quantum dot as our test case. This is still so small that in the presence of a magnetic field (the electron system being polarized) an essentially exact result can be obtained for several low-energy many-body states. The six electron system is the smallest size where the classical Wigner molecule has two nearly degenerate isomers [19], and as we shall see, the interplay of these isomers leads to interesting features in the many-body spectrum, as first pointed out by Maksym [7].

In the CI computations we use the Darwin-Fock states (with frequency $\omega_{\text {eff }}$ ) as our single particle basis. For the smallest fields, where the ground state is the MDD, it was essential to include single particle states from several Landau levels. The Fock states included were chosen with the help of a cut-off procedure: The matrix dimension was restricted to about 30000, which amounts to including about 50 Fock states. In the high-field case, corresponding to filling factor $1 / 3$, the single particle basis was restricted to the LLL, and a complete CI was used with single particle states up to $\ell=20$. (Note that the $q=3$ Laughlin state, to be discussed below, only includes single particle states up to $\ell=15$ for six electrons).

We calculated the many-body spectra for three different values of the magnetic field $(b=0.9,1.2$ and 2.5 in atomic units, corresponding approximately to filling factors $1,2 / 3$ and $1 / 3$, respectively). The strength of the external confinement was $\omega_{0}=0.34435$ a.u. (corresponding to a $2 \mathrm{D}$ electron density at the equilibrium density, $r_{s}=1.51$ a.u.). The many-body spectra are shown in Figs. 1 and 2. For each field strength we show the original spectrum (Fig. 四) and a spectrum displaying the interaction energy, where for each angular momentum value the center of mass contribution $L \hbar \omega_{\text {eff }}$ has been substracted (Fig. 2).

The results show that the overall structure of the spectrum is independent of the magnetic field, the role of which is mainly to tilt the spectrum so that the minimum energy will be at a finite angular momentum [20]. The spectrum starting from $L_{1}=15$ is nearly identical for the field strengths 0.9 and 1.2 and very similar to the spectrum corresponding to the strong field $b=2.5$ after the angular momentum $L_{3}=45$.

\section{TRIAL WAVEFUNCTIONS}

In order to get some understanding of the microscopic structure of the yrast states, it is useful to study them in terms of trial wavefunctions.

At angular momenta $L_{q} \equiv q N(N-1) / 2$ (with $q$ an odd integer), corresponding to filling fraction $\nu=1 / q$, an obvious guess for the ground state is the Laughlin wavefunction [8],

$$
\psi_{L}(q)=\prod_{i<j}^{N}\left(z_{i}-z_{j}\right)^{q} e^{-q \sum_{k}\left|z_{k}\right|^{2} / 4},
$$

where $z_{k}=\sqrt{m \omega_{\text {eff }} / q}\left(x_{k}+i y_{k}\right)$ are the complex particle coordinates. 
For $q=1$ this wavefunction is a Slater determinant of LLL single particle states, $\eta_{\ell} \propto$ $z^{\ell} \exp \left(-q z_{k}^{2} / 4\right)$, corresponding to angular momenta from $\ell=0$ to $\ell=N-1$. It has been shown that the wavefunction (1) describes very well the exact many-body state when the angular momentum corresponds to $q=3$ or larger $q$-values, both in a spherical geometry (which mimicks a homogeneous state) [21] and for a disk geometry with "open" boundary conditions [8,22]. In the case of $q=1$ the Laughlin wavefunction reduces to the (restricted) Hartree-Fock approximation which, as we shall see, is not a quantitatively exact description to the state for $N=6$, as the latter contains significant contributions from higher Landau levels.

For angular momenta above those described by the Laughlin wavefunction, $L=L_{q}+M$, ansatz wavefunctions can be constructed by multiplying the Laughlin-Jastrow factor by a fully symmetric polynomial in the $z_{k}$ of order $M$. In this way, the good correlations of the Jastrow factor are still built in. For $M=1$, the only way of doing this is multiplying by $\sum z_{k}$. This is just a center-of-mass excitation of the Laughlin state. (In a harmonic confinement the center of mass motion separates from the internal motion.) For $2 \leq M \leq N$ we propose

$$
\psi_{L B P}\left(L_{q}+M\right)=\mathcal{S} \prod_{i}^{M}\left(z_{i}-z_{0}\right) \psi_{L}(q)
$$

where the operator $\mathcal{S}$ symmetrizes the product, and $z_{0} \equiv \sum z_{k} / N$ denotes the center of mass coordinate.

The first, symmetric part of this wavefunction was introduced by Bertsch and Papenbrock [12] to describe the ground state of a weakly interacting, rotating Bose condensate for angular momentum up to the "unit vortex" state, i.e. $L \leq N$. In their case of a delta function interaction, the Bertsch-Papenbrock wavefunction has been proven to be exact [23] 25]. Here, we combine it with the Laughlin-Jastrow factor to construct a (fully antisymmetric) wavefunction that interpolates between the Laughlin state $L=L_{q}$ and the "quasihole" state $L=L_{q}+N$. Note that, indeed, for $M=N$ Eq.(2) just takes the form of the wavefunction proposed by Laughlin [5], for a quasihole centered at $z_{0}$.

We have tested the applicability of the Laughlin-Bertsch-Papenbrock (LBP) wavefunction (2) in the present case of six Coulomb-interacting electrons in a harmonic potential.

Table 1 gives the calculated overlaps of this wavefunction with the exact wavefunction starting from $L_{1}=15$ and from $L_{3}=45$. In the first case we determined only the values for $L_{1}+5$ and $L_{1}+6$ which are related to the localization of electrons (see next section). The LBP wavefunction seems to be a fairly good approximation also for the fermion case, especially for $q \geq 3$; the reason that the overlaps for $L=20,21$ are relatively poor, is that the Jastrow factor itself is not a very good approximation to the exact solution at $L=15$. As discussed previously, this is due to contributions from higher Landau levels in the exact wavefunction. If we perform an exact diagonalization for $q=1$ but restricted to the LLL, these latter overlaps will be much better (1.00, 0.96 and 0.99 for $L=15,20$ and 21, respectively).

In Table 1 we also give the most important single particle configuration of the CI calculation and its amplitude. The MDD is strongly dominated by only one configuration while the $L_{3}$ case is much more dispersed. We have argued that the Laughlin-Bertsch-Papenbrock ansatz cannot be accurate after the MDD; nevertheless, the most important configuration is consistent with the qualitative picture given by the LBP ansatz: For each additional 
quantum of angular momentum, one particle is pushed outwards from the center of the dot [3.

There is another class of trial wavefunctions [1], based on the idea of mapping strongly interacting electrons (or bosons) onto weakly interacting composite fermions by attaching an even (odd) number of flux quanta to each particle. These so-called Jain states have proven very successful in describing yrast states in quantum dots [10,26,27, as well as weakly interacting rotating Bose condensates [15,16]. Especially, the method explains the main features of the yrast spectra already in the noninteracting case, as illustrated in Fig. 2, and (in the interacting case) gives excellent overlaps with exact wavefunctions. However, the Jain construction involves technical steps (formally constructing states in higher Landau levels and subsequently projecting down to the LLL in a not uniquely defined way), which generally make it hard to visualize the qualitative features of the state. For this reason we choose to study the LBP ansatz which might not be as accurate as the Jain construction, but which in a very intuitive way "interpolates" between the Laughlin state $L_{q}$ and the 'quasihole' state $L_{q}+N$ and in a transparent way links the many-body spectrum to Wigner crystallization.

\section{LOCALIZATION OF ELECTRONS AND EDGE RECONSTRUCTION}

The original spectra of Fig. 11 show energy minima (cusps) at angular momenta 15, 20, 25, $30(b=0.9$ and 1.2$)$ and at 45, 50, 55, $60(b=2.5)$. This can be understood by localization of electrons in a five-fold ring (with one atom at the center). A rigid rotation of a ring of $N$ electrons can only have angular momenta $L=n N$ if $N$ is odd and $L=(n+1 / 2) N$ if $N$ is even ( $n$ is an integer) [28]. Other angular momentum values can only be a result of center of mass excitations or as a combination of the rigid rotation with vibrational modes. The analysis of rotation-vibration spectra was found to be successful also in studying localization of non-polarized electrons in quantum rings [29].

The spectra also show markedly low energies when $L$ is $21,27,51,57$, corresponding to a six-fold symmetry. Classically the six-electron system has also a stable geometry of a six-fold ring [19, which is only slightly higher in energy than the five-fold ring. In the special points $L=15$ and $L=45$ the quantum state must be a combination of these two structures. If the angular momentum is increased by 5 , the solution collapses into the five-fold symmetry, and if it is increased by 6 the solution collapses into the six-fold ring.

Cusps of the type seen here, have previously been noticed for a few-electron system and discussed in terms of particle localization [20] and symmetry 30,31 as well as composite fermions [10]. Here we will first use the exact solutions for the six electron dot to test our qualitatively simple picture of localized electrons.

Figures 3 and 4 show the radial densities of the CI computations for different values of the angular momentum at the yrast line. Clearly the densities for $L=20,25,45$ and 50 are consistent with the five-fold ring, having one particle in the center, while the results for $L=21,27,51$ have only one ring of electrons.

It is illustrative to study the electron localization also in terms of analytic trial wavefunctions. The ' $N$-particle correlation function' is simply defined as the square of the wavefunction 


$$
\rho\left(z_{1}, z_{2}, \cdots, z_{N}\right)=\left|\psi\left(z_{1}, z_{2}, \cdots, z_{N}\right)\right|^{2} .
$$

Laughlin [8] showed that in the case of Eq. (11) finding the maximum of the correlation function is equivalent to finding the equilibrium electron positions in a classical one-dimensional plasma, where the electrons interact via a repulsive logarithmic potential and are confined by a harmonic trap. In accordance with the above discussion, for six particles and $L=15$ or 45 , there are two local minima corresponding to the five- and six-fold geometries, respectively (the former being the lowest).

Moreover, Laughlin showed that the optimal geometry is independent of the exponent $q$ of the wavefunction. Scaling the complex coordinates with $q$ as we did in Eq. (1), also the distances between the localized electrons are independent of $q$. The Laughlin wave function for even $q$-values describes well rotating Bose condensates [15,16]. Consequently, at large angular momenta the statistics of the particles do not play any role in the internal correlation of the particles. In fact, this picture is also fairly independent of the actual interaction potential, since the Laughlin state is an excellent approximation for long-range Coulomb interactions as well as zero-range interactions [5].

The localization becomes stronger when $q$ increases, making the Laughlin state gradually more similar to a crystal [5]. This can be measured by the sharpness of the maximum of $\rho$. It is easy to show that around a maximum correlation

$$
\left(\frac{\partial^{2}}{\partial x_{1}^{2}}+\frac{\partial^{2}}{\partial y_{1}^{2}}\right) \rho\left(z_{1}, z_{2}^{0}, \cdots, z_{N}^{0}\right)_{z_{1}=z_{1}^{0}}=-2 q \rho\left(z_{1}^{0}, z_{2}^{0}, \cdots, z_{N}^{0}\right),
$$

where $z_{i}^{0}$ are the coordinates of the maximum. This result explains why the density of the MDD is nearly constant, while the density corresponding to $q=3$ has sharper maxima consistent with a configuration of electrons localized in a five-fold geometry.

Also, the exact many-body spectra of Fig. 11 show clearly that the five-fold ring becomes more dominating after $L_{3}$ than after $L_{1}$, as it drops below the six-fold one for all $L>L_{3}$.

Our analytical ansatz of Eq. (2) allows for the study of localization of electrons at any angular momentum after any $L_{q}$. Figure 5 shows the positions of electrons at the correlation maxima of Eq. (22) for $L=45,47,48,49,50$, and 51 ( $L=46$ is an uninteresting center of mass excitation). The five-fold ring at 50 and the six-fold ring at 51 are reproduced as anticipated. In general, the outer ring consists of as many electrons as is the order of the polynomial multiplying the Laughlin wavefunction.

Note that the geometries are independent of $q$; for example they would be the same for bosons at $L$ values ranging from 30 to 36 . (Or, in fact, for non-integer $q$, which corresponds to anyons, i.e. particles obeying fractional statistics, in the LLL [32])

For systems containing a large number of electrons, it is not possible to perform exact calculations of the yrast spectra. However, in the region where the particles localize, simple arguments can be used to predict properties of the yrast spectra. The localization in the Laughlin state corresponds to the classical plasma with logarithmic interactions. The ground state positions of the electrons are very similar to those obtained with $1 / r$ interactions, which have been analyzed by Schweigert and Peeters [33]. In finite systems the resulting Wigner molecules are characterized by rings of electrons (as seen already in the case of six electrons). Figure 6 shows as an example the 18 electron geometry. If the Laughlin state is again multiplied by a polynomial as in Eq. (2), it follows that especially favourable are 
cases where the order of the polynomial equals the number of electrons in the outermost ring. This case is demonstrated in Fig. 6 which shows that when the angular momentum is increased by 11, i.e. the number of electrons in the outer ring, the only effect is the expansion of this outer ring.

The above analysis suggests that starting from the Laughlin state, the next low-energy state (cusp) is obtained at angular momentum $L_{q}+n$ where $n$ is the number of electrons in the outermost ring of the corresponding classical electron system. In the case of the maximum density droplet this corresponds to the expansion of the so-called Chamon-Wen [4] edge from the MDD. Indeed the number of electrons in the Chamon-Wen edge as calculated by Reimann et al. [1] for $N=20$ is in agreement with the number of classical electrons in the outermost ring. Increasing the angular momentum further by the number of electrons in the next ring causes also that ring to expand, and again an energy minimum is expected.

The present scheme thus provides microscopic trial wavefunctions describing the edge reconstruction of small quantum Hall droplets. For the special case of the MDD $(q=1)$ a similar trial wavefunction was recently proposed by Goldmann and Renn [2].

The expansion of the Chamon-Wen ring also increases the electron localization. This can be demonstrated by studying the decrease of the $N$-particle correlation when an electron is moved out from the position of the maximum correlation (keeping all other electrons in place). Figure 7 shows the decrease of the correlation as a function of the electron displacement (angular average) for the six electron dot for the MDD $(L=15)$, for the Chamon-Wen state $(L=20)$ and for the $q=3$ Laughlin state $(L=45)$ calculated using the LBP ansatz Eq.(2). It is clear that in the Chamon-Wen edge state the electron localization is increased as compared to the MDD. The difference between the $L=45$ and $L=15$ states in Fig. 7 is in agreement with Eq. (田).

Let us finally comment on the case $L=L_{q}+N$. Our ansatz (2) then has the same form as that proposed by Laughlin [8] to describe the fractionally charged quasihole excitations of large quantum Hall systems. In these systems, the quasihole is the lowest-lying bulk excitation. The finite size of the quantum dot, with no clear distinction between bulk and edge, allows also for low energy excitations where one surface layer (of localized electrons) at a time is excited. As we have argued, the classical interpretation of these excitatitons is that the outer ring is rigidly rotating. The lowest energy excitation then corresponds to angular momentum increase which equals the number of electrons in the outermost ring. This is already seen in the six electron case where the low energy excitations after the $L_{3}=45$ state have $L=50,55$ etc in accordance with the 5 electrons in the ring around the center atom.

\section{CONCLUSIONS}

We have demonstrated that the localization of electrons in a strong magnetic field can be seen in the structure of the many-body spectrum. The many-body yrast states following each Laughlin state can be approximated with simple trial wavefunctions similar to those originally suggested by Bertsch and Papenbrock [12] for rotating Bose condensates.

In a quantum dot the internal structure of the Laughlin state corresponds to localization of electrons in consecutive rings. The Laughlin-Bertsch-Papenbrock wavefunction illustrates that the Chamon-Wen edge reconstruction can be seen as an expansion of the outermost ring. Since the Laughlin and LBP wavefunctions are good approximations for fermions as 
well as bosons and both for long- and short range interactions, it seems that the internal geometry of the localized particles in a high rotational state depends only on the number of particles in the harmonic confinement. 


\section{REFERENCES}

[1] S.M. Reimann, M. Koskinen, M. Manninen, and B. Mottelson, Phys. Rev. Lett. 83, 3270 (1999).

[2] E. Goldmann and S.R. Renn, Phys. Rev. B 60, 16611 (1999).

[3] A.H. MacDonald, S.R.E. Yang, and M.D. Johnson, Aust. J. Phys. 46, 345 (1993).

[4] C. de C. Chamon and X.G. Wen, Phys. Rev. B 49, 8227 (1994).

[5] The Quantum Hall Effect, edited by E. Prange and S.M. Girvin (Springer-Verlag, New York, 1990)

[6] H.-M. Müller and S.E. Koonin, Phys. Rev. B 54, 14532 (1996).

[7] P.A. Maksym Phys. Rev. B 53, 10871 (1996).

[8] R.B. Laughlin, Phys. Rev. Lett. 50, 1395 (1983).

[9] G. Dev and J.K. Jain, Phys. Rev. B 45, 1223 (1992).

[10] J.K. Jain and T. Kawamura, Europhys. Lett. 29, 321 (1995).

[11] For a review, see J.K. Jain and R.K. Kamilla, in Composite Fermions: A Unified View of the Quantum Hall Effect, edited by O. Heinonen (World Scientific, River Edge, NJ 1998)

[12] G.F. Bertsch and T. Papenbrock, Phys. Rev. Lett. 83, 5412 (1999).

[13] N.K. Wilkin, J.M.F. Gunn and R.A. Smith, Phys. Rev. Lett. 80, 2265 (1998).

[14] N.K. Wilkin and J.M.F. Gunn, Phys. Rev. Lett. 84, 6 (2000).

[15] N.R. Cooper and N.R. Wilkin, Phys. Rev. B 60, R16297 (1999).

[16] S. Viefers, T.H. Hansson, and S.M. Reimann, Phys. Rev. A 62, 053604 (2000).

[17] V. Fock, Z. Phys. 47, 446 (1928).

[18] C.G. Darwin, Proc. Camb. Phil. Soc. 27, 86 (1930).

[19] F. Bolton and U. Rössler, Superlattices and Microstructures 13, 139 (1992).

[20] P.A. Maksym and T. Chakraborty, Phys. Rev. Lett. 65, 108 (1990).

[21] F.D.M. Haldane and E.H. Rezayi, Phys. Rev. Lett. 54, 237 (1985).

[22] S.A. Trugman and S. Kivelson, Phys. Rev. B 31, 5280 (1985).

[23] A.D. Jackson and G.M. Kavoulakis, Plys. Rev. Lett. 85, 2854 (2000).

[24] R.A. Smith and N.K. Wilkin, Phys. Rev. A 62, 061602 (2000).

[25] T. Papenbrock and G.F. Bertsch, Phys. Rev. A 63, 023616 (2001).

[26] B. Rejaei, Phys. Rev. B 48, 18016 (1993).

[27] C.W.J. Beenakker and B. Rejaei, Physica B 189, 147 (1993).

[28] M. Tinkham, Group Theory and Quantum Mechanics (McGraw-Hill, New York 1964) explains the two-electron case which can be easily extended to an $N$-electron ring.

[29] M. Koskinen, M. Manninen, B. Mottelson, and S.M. Reimann, Phys. Rev. B 63, 205323 (2001).

[30] W.Y. Ruan, Y.Y. Liu, C.G. Bao, Z.Q. Zhang, Phys. Rev. B 51, 7942 (1995).

[31] C.G. Bao, Phys. Rev. Lett. 79 (1997).

[32] T.H. Hansson, J.M. Leinaas and S. Viefers, Nucl. Phys. B 470, 291 (1995).

[33] V. Schweigert and F.M. Peeters, Phys. Rev. B 51, 7700 (1995). 


\section{FIGURES}

FIG. 1. Calculated many-body states as a function of angular momentum for three different values of the external magnetic field. The lowest energy states corresponding to a five-fold (six-fold) symmetry are connected with a dashed (dotted) line.

FIG. 2. Many-body states of Fig. 11 but with the center of mass excitation energy $L \hbar \omega_{\text {eff }}$ substracted. The structure of the yrast line predicted by the Jain construction is shown as dashed lines.

FIG. 3. Radial density distributions of the exact many-body states in a six electron quantum dot at a magnetic field of $b=2.5$ a.u. $L$ is the total angular momentum.

FIG. 4. Radial density distributions of the exact many-body states in a six electron quantum dot at a magnetic field of $b=1.2$ a.u. $L$ is the total angular momentum.

FIG. 5. Geometry of the 'Wigner molecule' of six electrons for different values of the angular momentum $L$, calculated from the trial wavefunction Eq.(2).

FIG. 6. Positions of electrons in an 18-electron dot at the maximum of the square of the wavefunction (2) for $L=q N(N-1) / 2=153 q$ (filled circles) and $L=153 q+11$, 11 being the number of electrons in the outermost ring.

FIG. 7. The decrease of $N$-particle correlation when one electron is moved out from the

maximum position. The distance is given as percentage of the nearest neighbour distance. Solid line $L=15$, dashed line $L=20$, dotted line $L=45$. The dot has six electrons. 


\section{TABLES}

TABLE I. Absolute values of the overlaps between the exact wavefunction and the approximate wavefunction, Eq. (2), for different values of the angular momentum $L$. MIC is the most important single particle configuration as occupation numbers of the LLL states starting from $\ell=0$. The last column gives the amplitude of the MIC.

\begin{tabular}{lccc}
\hline \hline$L$ & $\left|\left\langle\psi_{B P} \mid \psi\right\rangle\right|$ & MIC & amplitude of MIC \\
\hline 15 & 0.88 & 1111110000000000 & 0.877 \\
20 & 0.87 & 1011111000000000 & 0.743 \\
21 & 0.90 & 0111111000000000 & 0.833 \\
25 & & 1001111100000000 & 0.634 \\
27 & & 0011111100000000 & 0.655 \\
\hline 45 & 0.99 & 1000000111110000 & 0.373 \\
47 & 0.94 & 1000000111100100 & 0.180 \\
48 & 0.96 & 1000000110111000 & 0.184 \\
49 & 0.94 & 1000000101111000 & 0.230 \\
50 & 0.93 & 1000000101110100 & 0.290 \\
51 & 0.96 & 0100000011111000 & 0.257 \\
\hline \hline
\end{tabular}




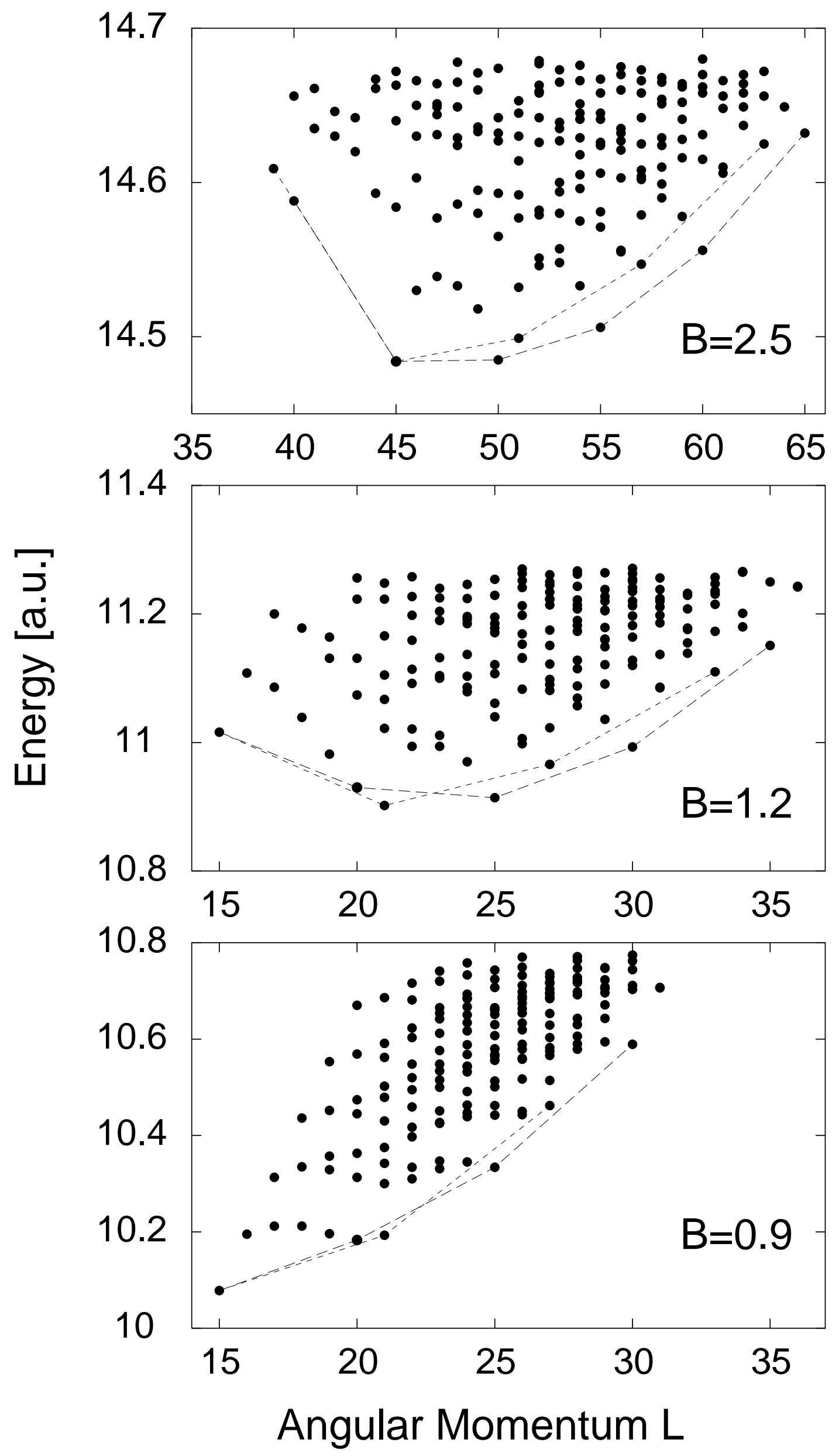




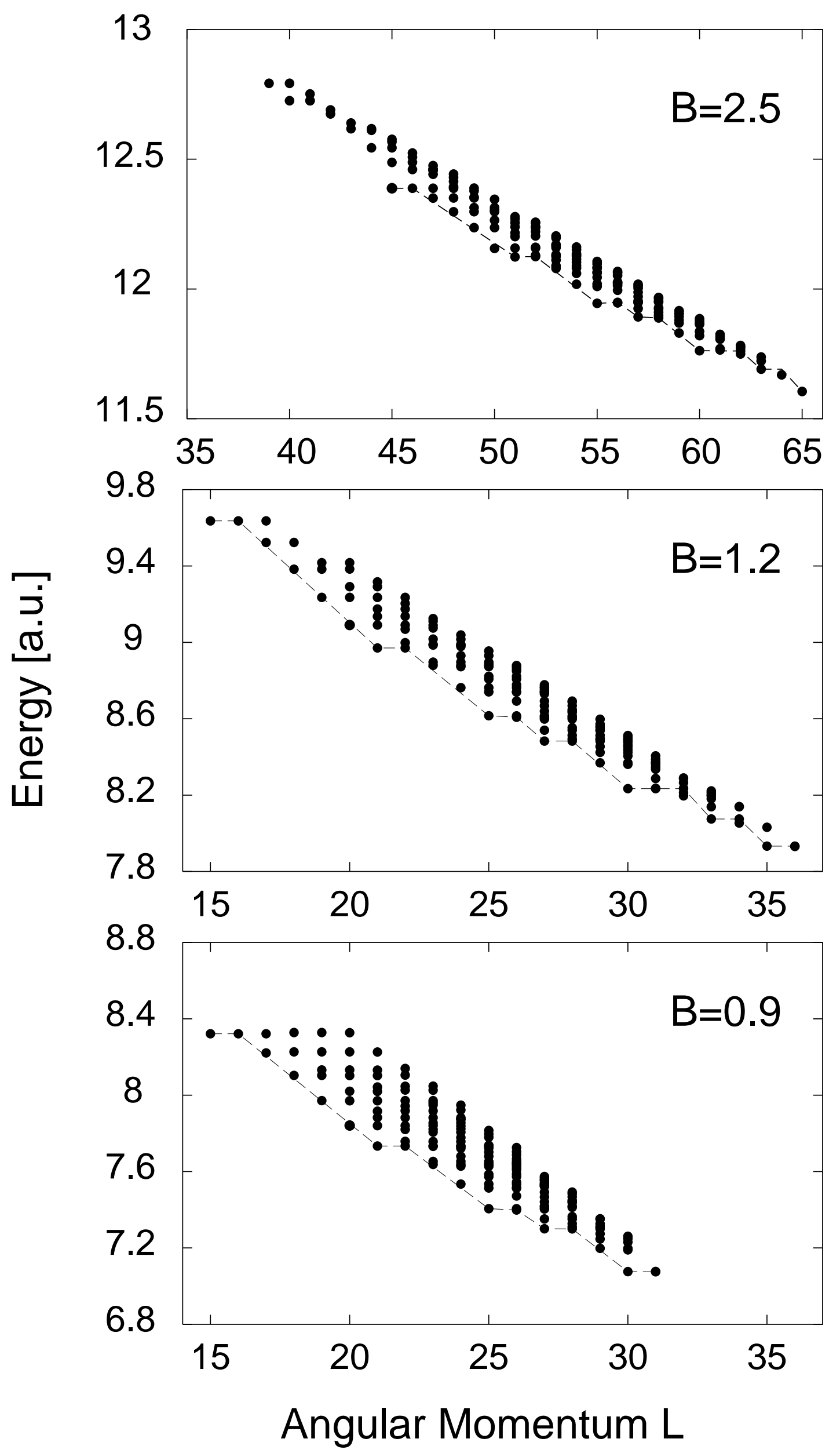




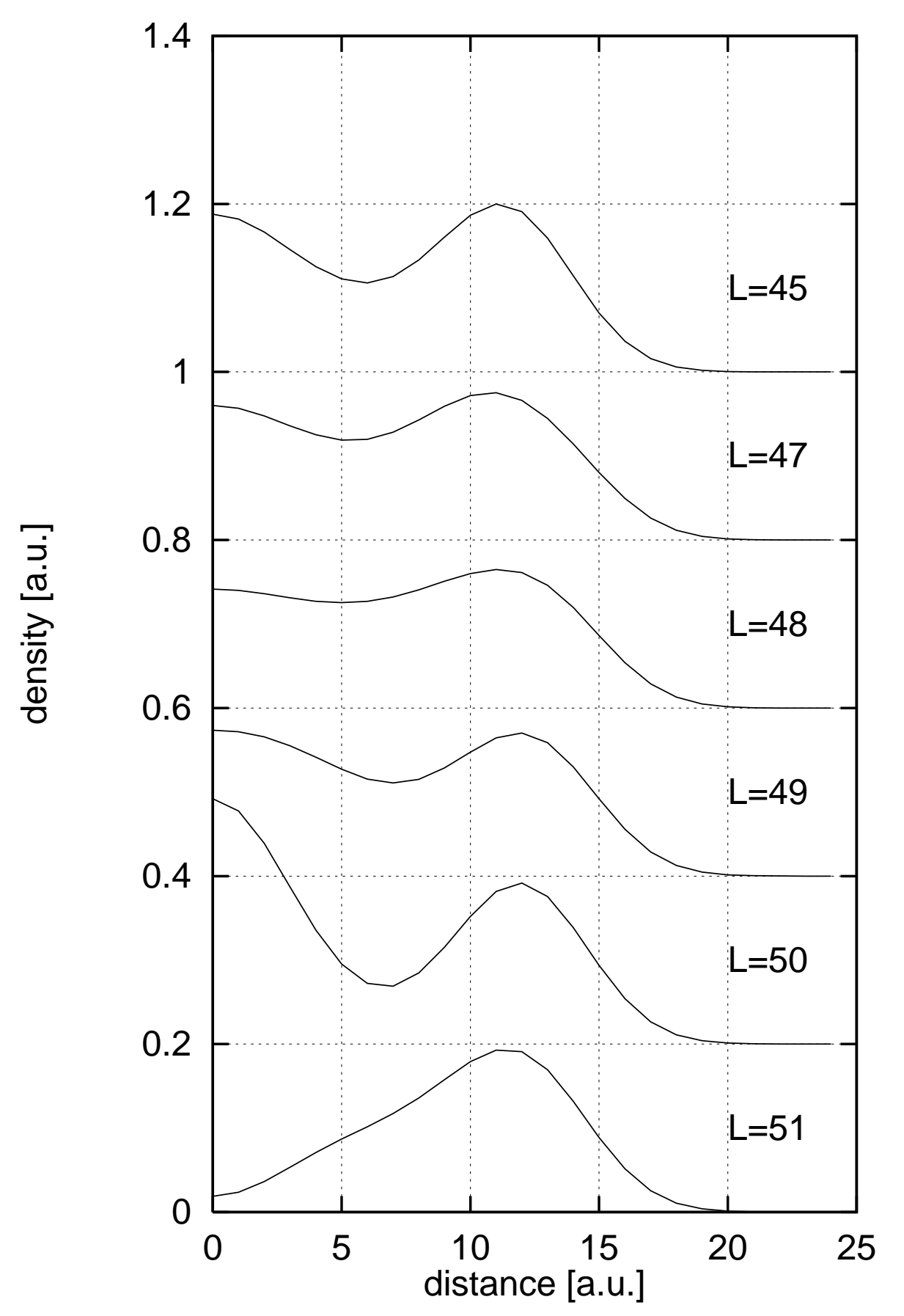




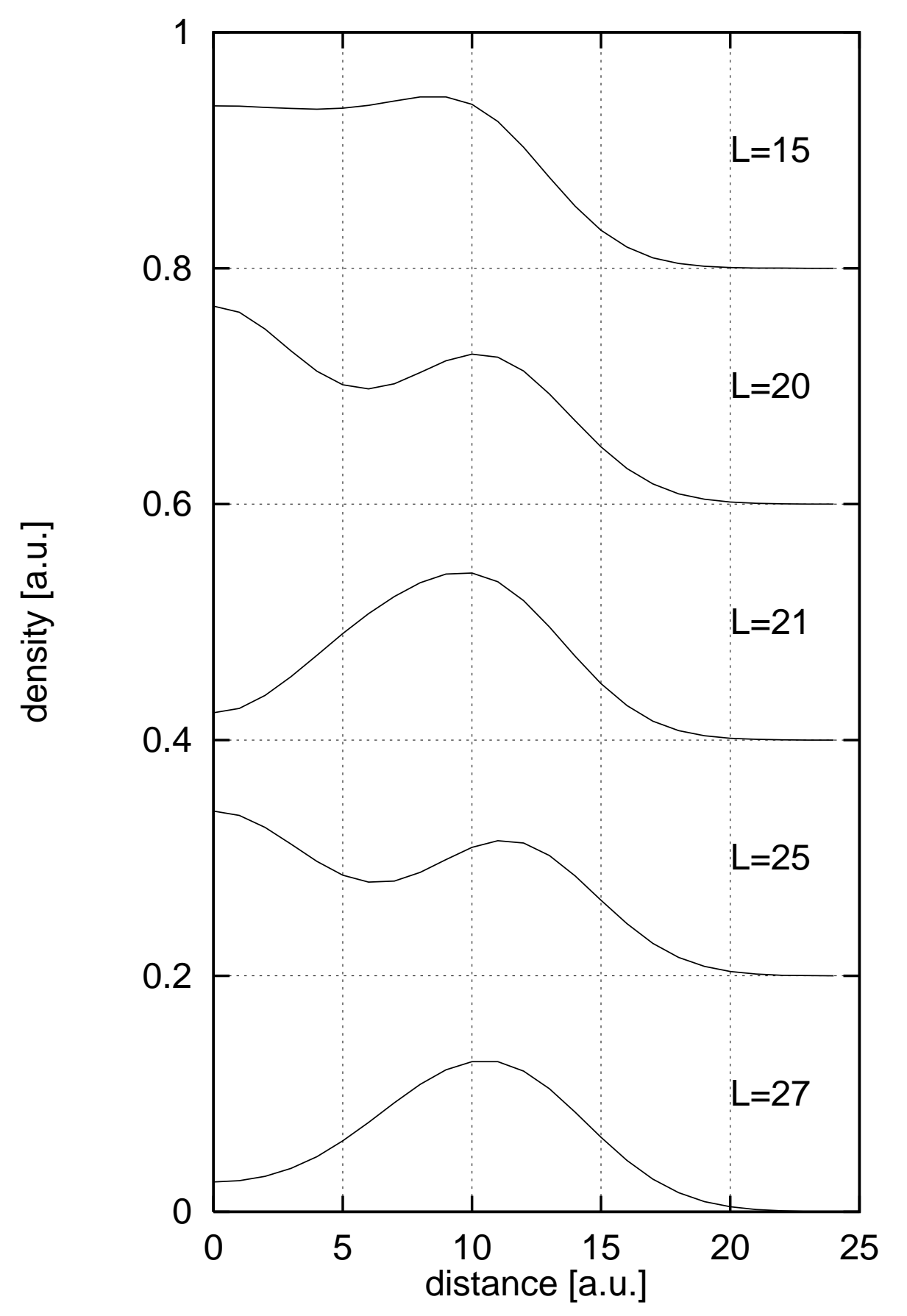




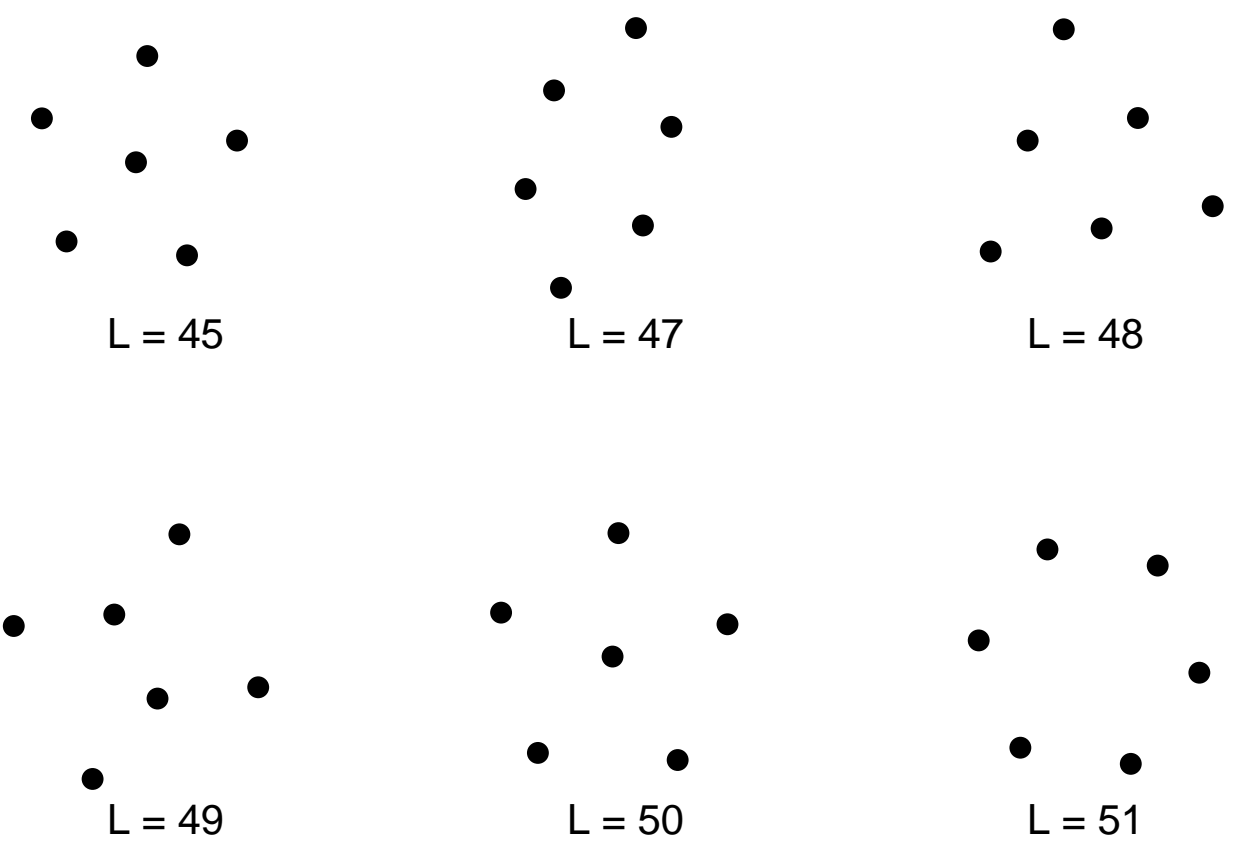




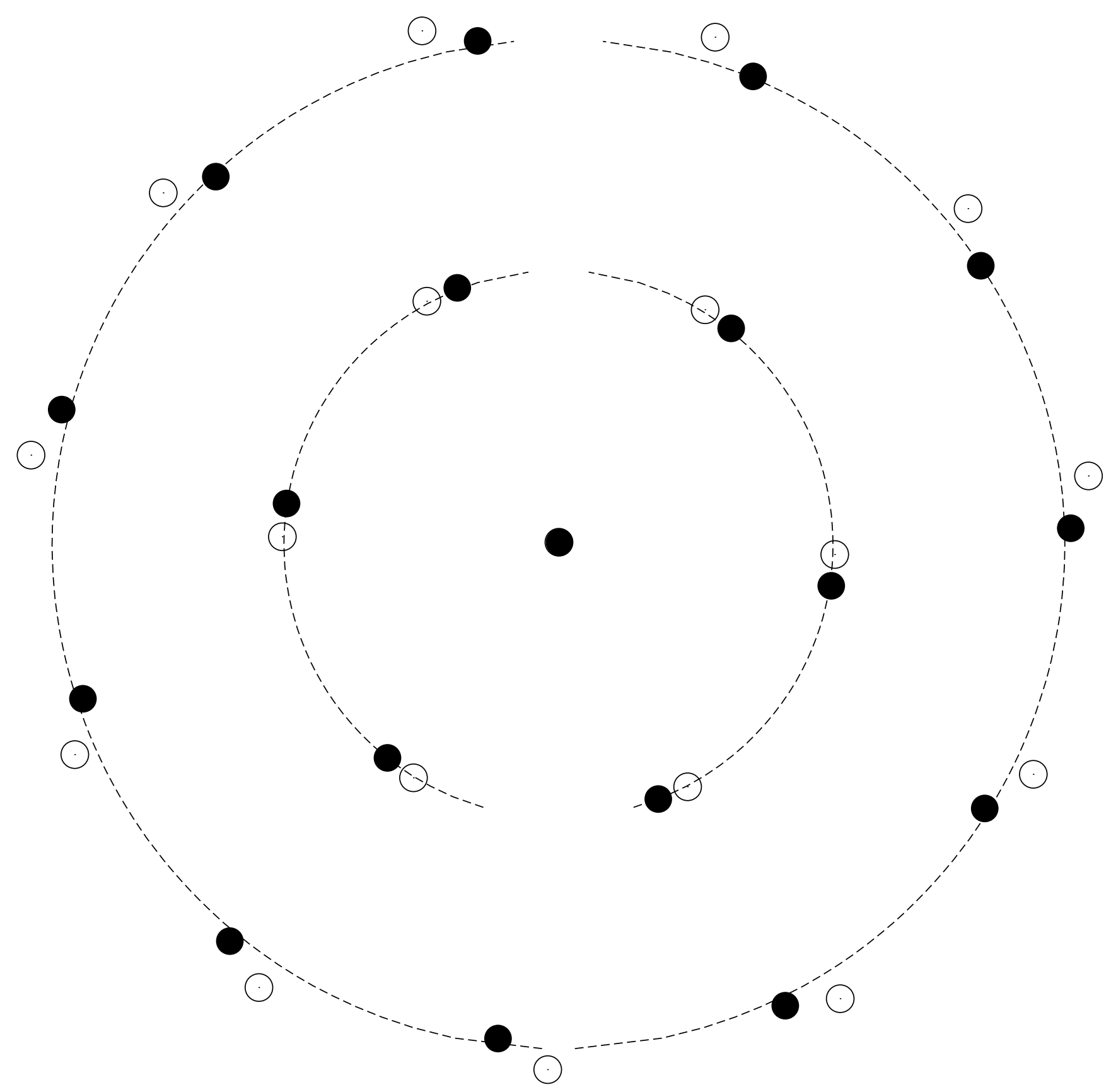




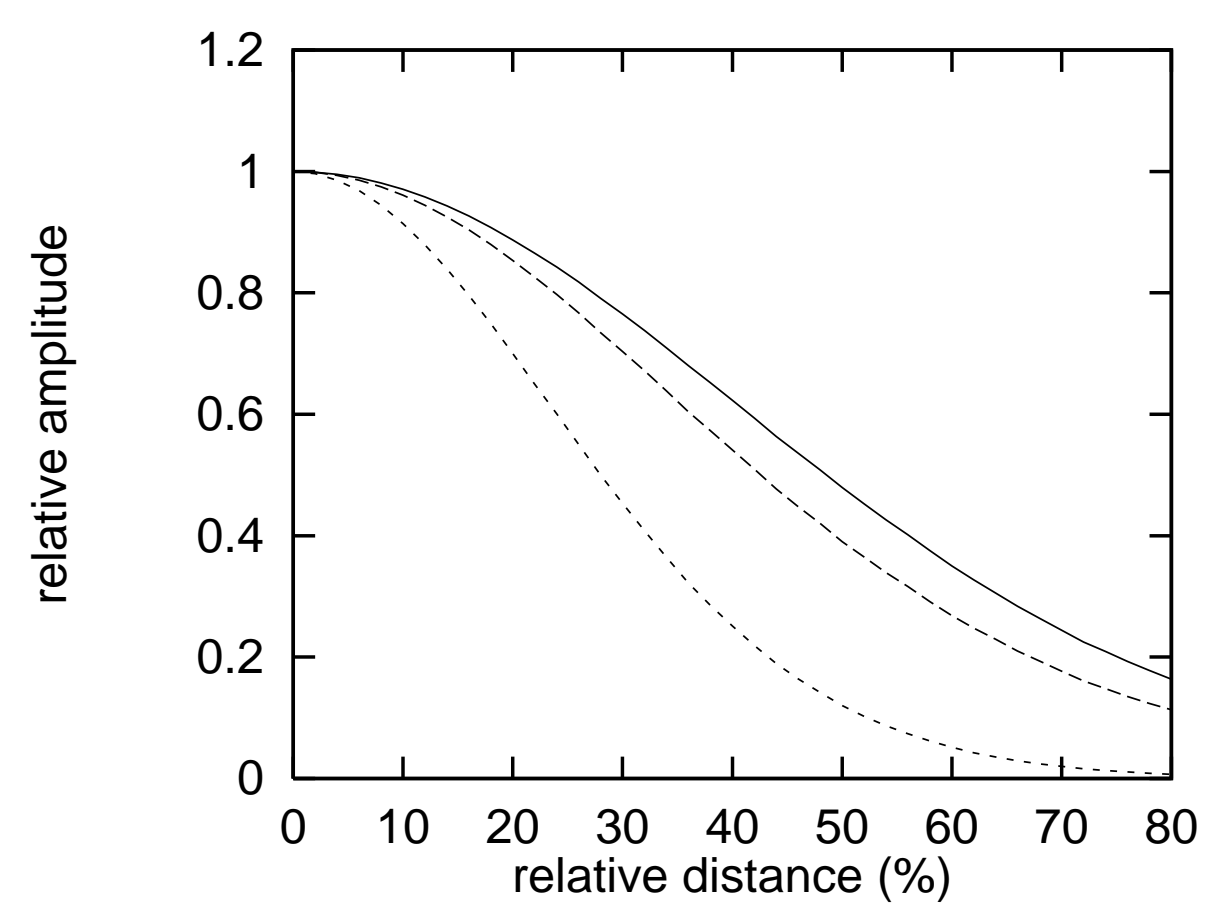

